\title{
EFFICIENCY OF CANCER TREATMENTS: IN SILICO EXPERIMENTS
}

\author{
Elena Piretto ${ }^{1,2}$, Marcello Delitala $^{1, *}$ and Mario Ferraro ${ }^{3}$
}

\begin{abstract}
Despite the advances in the formulation of different therapies to fight cancer, the design of successful protocols is still a challenging problem. In order to provide some indications on the effectiveness of medical treatments, results from in silico experiments are presented based on a mathematical model comprising two cancer populations competing for resources and with different susceptibilities to the action of therapies. The focus is on the outcome of protocols in which the total dose can be administered with different time distributions. An efficiency index is proposed to quantify the effectiveness of different protocols. Simulations show that a standard dose chemotherapy is effective when the sensitive clone has a marked competitive advantage, whereas its outcome is much worse when a resistant clone emerges; obviously combinations of immune and chemotherapy work better. These results, in accord with previous finding reported in the literature, stress the importance to take into account competitive interactions among cancer clones to decide which therapeutic strategy should be adopted. However, it is not just the efficiency that changes in these different configurations of clonal composition and therapy timing. A general rule seems to emerge: when evolutionary pressures are strong, the best protocols entail and early starting of the treatment, whereas, on the contrary, when interactions among clones are weak, therapy should start later. Finally the model has been adapted to investigate the relative efficiency of different protocols, by using data reported in literature regarding experiments with breast cancer cells.
\end{abstract}

Mathematics Subject Classification. 92B05, 92D25.

Received December 11, 2018. Accepted July 11, 2019.

\section{INTRODUCTION}

In organisms cancer does not appear as a single species but rather as a mixture of different populations [35], and this heterogeneity is a major factor in cancer drug resistance, see e.g. [30]. Even though a therapy can eliminate a cancer type, one or more resistant variants of the tumor population may exist, driving to the resurgence of treatment-refractory disease [13].

The solution to this problem lies, at least in theory, in therapies that combine different agents, with specific actions, thus increasing the likelihood of synergistic antitumoral effects [8]. The design of combined protocols is

Keywords and phrases: Cancer modelling, population dynamics, therapeutic protocols, breast cancer.

1 Department of Mathematical Sciences, Politecnico di Torino, Italy.

2 Department of Mathematics, Universitá degli Studi di Torino, Italy.

3 Department of Physics, Universitá degli Studi di Torino, Italy.

* Corresponding author: marcello.delitala@polito.it 
a challenging problem and, specifically, the optimal dosing and timing in the combination of chemotherapy and immunotherapy is still an open issue [33].

Indeed, a preliminary question is to identify the targets of protocols' optimization. Different factors can be thought of: for instance, the cancer load at the end of the therapies should be as small as possible, but of course also the total cancer load during the therapy period must not grow unchecked. Furthermore, it is clearly important to control cancer clones resistant to treatment.

In previous papers $[26,27]$, it has been shown that clonal composition plays a crucial role in determining outcomes of cancer treatments. More specifically, a model has been proposed, based on the theory of interacting populations, comprising two cancer populations competing for resources and with different susceptibilities to the action of immune system cells and therapies. Results have shown that the outcomes of treatment depend on the relative strength of the competitive interactions between clones.

In the present paper, these findings provide the background for an analysis of treatments efficiency for protocols characterized by different schedules of drug administration. In particular, here we aim to determine the best time course for a chemotherapy with a fixed total dose for different competitive strength of cancer clones. The goodness of the results is assessed by a suitable index. This approach is then extended to the case of combined treatments, i.e. chemo- and immunotherapy. Finally, results of the simulations are compared with data of the literature.

\section{THE MODEL}

Cancer models based on differential equations form a vast body of literature: see, among others [9, 11, 12]. There exist models considering tumorigenesis in greater detail than is done here, for instance $[4,7,34,36]$; as concerns the interaction of cancer with the immune system, $[3,9,20]$, modelling of therapies, $[14,31]$ and more specifically on drug resistance to therapies, [5, 15, 21]. Extensive bibliographic references can be found in the review papers $[2,10]$. The model used here, proposed in [26], has been developed in the framework of population dynamics, [25], to describe the evolution of cancer and immune system. Tumor heterogeneity is taken into account by considering two cancer clones, or populations, with $x_{1}, x_{2}$ denoting ambiguously both the cancer type and the corresponding number of tumor cells for each clone. The number of immune cells is represented by $z$. Evolution of cell populations is determined by three basic elements: proliferation, predation and competition for resources. Equations are as follows:

$$
\begin{aligned}
& \frac{\mathrm{d} x_{1}}{\mathrm{~d} t}=\underbrace{r_{1} x_{1}-\frac{r_{1}}{K_{1}} x_{1}^{2}}_{\text {proliferation }}-\underbrace{\frac{b_{12}}{K_{1}} x_{1} x_{2}}_{\text {competition }}-\underbrace{\frac{c_{1}}{K_{1}} x_{1} z}_{\text {predation }} \underbrace{z-g_{1}(t) x_{1}-\frac{h(t)}{K_{1}} x_{1} z}_{\text {therapies }}, \\
& \frac{\mathrm{d} x_{2}}{\mathrm{~d} t}=r_{2} x_{2}-\frac{r_{2}}{K_{2}} x_{2}^{2}-\frac{b_{21}}{K_{2}} x_{1} x_{2}-\frac{c_{2}}{K_{2}} x_{2} z-g_{2}(t) x_{2}-\frac{h(t)}{K_{2}} x_{2} z, \\
& \frac{\mathrm{d} z}{\mathrm{~d} t}=\underbrace{\beta z\left(1-\frac{z}{H}\right)}_{\text {proliferation }}+\underbrace{\frac{\alpha_{1}}{H} x_{1} z+\frac{\alpha_{2}}{H} x_{2} z}_{\text {recognition }} .
\end{aligned}
$$

The model is described in greater detail in [26], where also stability analysis of stationary states is carried out. Here we present a short summary:

- Tumor clone $x_{1}$. Clone $x_{1}$ undergoes a logistic growth with reproduction rate $r_{1}$ and carrying capacity $K_{1}$, i.e. $K_{1}$ is the maximum value $x_{1}$ can take. Development of $x_{1}$ is constrained by the competition with clone $x_{2}$ (measured by the parameter $b_{12}$ ) and by the interaction with the immune system (parameter $c_{1}$ ).

- Tumor clone $x_{2}$. The equation for $x_{2}$ is analogous to the one for $x_{1}$ and the same considerations apply.

- Immune system $z$. It grows with a net rate $\beta$ and, in absence of tumor, it is limited by $H$. In presence of cancer, $z$ undergoes a clonal expansion weighted, respectively, by parameters $\alpha_{1}$, $\alpha_{2}$ which measure the ability of immune cells to detect and recognize cancer cells. 
- Chemotherapy. The effects of chemotherapy on the population $x_{i}$ are represented in the model by the term $g_{i}(t) x_{i}$ where $g_{i}$ takes into account the drugs kinetics in the organism, see [6]. This is equivalent to rewrite the growth term as $f_{i} x_{i}=\left(r_{i}-g_{i}(t)\right) x_{i}$.

- Immunotherapy. The action of the immune system can be enhanced by immunotherapy, whose effect is expressed by the term $h(t) / K_{i} x_{i} z, h(t)>0$ which is equivalent to define a new parameter $\kappa_{i}=c_{i}+h(t)$. The effect of the immunotherapy as an increased ability by the immune system to recognize and kill cancer cells has been proposed, for instance, in [36].

In the following cancer clone $x_{1}$ is supposed to be susceptible to chemotherapy, whereas $x_{2}$ is resistant, i.e. $g_{2}(t)=0 \forall t$. Moreover, $x=x_{1}+x_{2}$ denotes the total cancer load.

A detailed stability analysis of system (2.1) and the stationary points has been presented in [26] and it can be carried out with the standard methods of analysis. The main results are reported here in view of the discussion of Section 4. The analysis has been performed considering cancer populations before treatment, to identify conditions for the occurrence of different trends. Thus, what is of interest are not the stationary points, per se, but rather the correspondence between parameters and composition of the cancer population.

The stable points of biological interest correspond to the case of tumor eradication, survival of a single cancer clone (competitive exclusion) and coexistence of both cancer clones. Furthermore, it is assumed that, before therapy, both cancer clones escape eradication by the immune system; more specifically the focus is on two types of configurations of cancer populations, characterized by different asymptotic behaviours, namely coexistence of susceptible and resistant clones (heterogeneous tumors) and competitive exclusion of the resistant clone. Summing up:

- Coexistence $(C O E)$. Before therapy, $x_{1}$, the susceptible clone, is also the fittest, as it has a larger rate of growth and also a competitive advantage even though $x_{2}$ is not totally eliminated.

- Competitive exclusion (EC1). $x_{1}$ is the dominant clone. In this configuration $x_{1}$ increases its competitive advantage so that, asymptotically, $x_{2}$ is wiped out.

Different combinations of parameters give rise to different degrees of competition and, hence, to different levels of coexistence or, for $E C 1$ to different time courses for the disappearance of the weaker clone. Thus, to the $C O E$ configuration may correspond different equilibrium values of clones population, and, also, transients of $x_{1}, x_{2}$ in $C O E$ may be similar to those for a relatively weak competitive exclusion.

\section{MeAsuRES OF TREATMENT EFFICIENCY}

An usual measure of efficiency of treatment is given via the functional:

$$
H(u)=\alpha x(T)+\int_{0}^{T}(\beta x(t)+\gamma u(t)) \mathrm{d} t,
$$

where $x$ is the total cancer population, $T$ the duration of the therapy and $u$ is the time course of the treatment, see e.g. $[6,18,19]$. The optimal therapy $u^{*}$ is then the one which minimizes $H$, subjected to the constraint (represented by the last term in the integral) that the doses of the drugs administered to the patient must not be too high. Minimization of the functional $H$ can be obtained using standard methods of control theory. However the problem remains of how to choose the values of the parameters $\alpha, \beta$ and $\gamma$ that determine the weights of the factors in $H$. Furthermore, equation (3.1) fails to consider fact that a decrease of the total load $x$ can be misleading, if due just to a reduction of the sensitive clone $x_{1}$, in that it may mask an increase of the resistant species $x_{2}$.

Thus, a measure of the efficiency of a treatment should take into account not just the decrease of the total cancer load $x=x_{1}+x_{2}$ but also the trend of resistant cells, a critical factor in assessing how well a therapy works. An index of effectiveness depending on variation of the total load and of the number of resistant cells has been defined in [27] as follows. Denote by $x_{\text {pre }}, x_{\text {post }}$ the total cancer loads one day before and one day after the 
administration of the therapy and by $x_{2, \text { pre }} x_{2 \text {,post }}$ the corresponding values of $x_{2}$ (the resistant clones). Next define parameters $\epsilon$ and $\delta$ as

$$
\epsilon=\frac{x_{\text {pre }}-x_{\text {post }}}{x_{\text {pre }}}, \quad \delta=\frac{x_{2, \text { pre }}-x_{2, \text { post }}}{x_{\text {pre }}} .
$$

The upper bound of $\epsilon$ and $\delta$ is 1 ; they can take negative values, whereas, to combine them in a global parameter, they need to be non-negative. A transformation is then applied to $\epsilon, \delta$ by means of the sigmoid function and finally an efficiency index $\gamma$ is defined as

$$
\gamma=\frac{(1+\exp (-1))^{2}}{[1+\exp (-\epsilon)][1+\exp (-\delta)]}
$$

where the term $(1+\exp (-1))^{2}$ ensures that $\sup \gamma=1$.

The index $\gamma$ depends solely on the numerosity of $x_{1}$ and $x_{2}$ before and after the treatment, but it fails to take into account the cancer load during the therapy which also is important to assess the effectiveness of a therapy. In order to include this factor, a new index is proposed here. Let $x_{c}$ be the total load in absence of treatment (the control case): the measure of therapy efficiency then becomes

$$
I=\gamma\left(1-\frac{\int_{T_{i}}^{T_{f}} x(t) \mathrm{d} t}{\int_{T_{i}}^{T_{f}} x_{c}(t) \mathrm{d} t}\right)
$$

Obviously no index can fully capture the complexity involved in the results of medical treatments. The index $I$ is meant to give just some insight to the relative effectiveness of different therapies on therapeutic outcomes; in other words what matters here it is not the absolute value of $I$ but, rather, the relative values in the different cases.

\section{Simulations AND RESUlts}

In this section it is carried out a comparison of the results of various protocols, by the index $I$, characterized by different temporal distribution of the dose.

Parameters used in the simulations have been evaluated from data reported in the literature [7, 16], or, as in case of $b_{12}, b_{21}$, varied in an exploratory way. Table 1 reports their values. We focus on two types of cancer populations, characterized by different competition interactions and different asymptotic behaviours as highlighted in Section 2: Coexistence $(C O E)$ where $b_{12}=0.001$ and $b_{21}=0.098$, and Competitive exclusion $(E C 1)$ where $b_{12}=0.1$ and $b_{21}=0.5$.

Initial conditions are chosen as follow: cancer evolution starts with resistant population, $x_{2}$, small with respect to the susceptible, $x_{1}$, i.e. $x_{1}(0)=9 \times 10^{4}, x_{2}(0)=4 \times 10^{4}$, and the immune cells are $z(0)=5 \times 10^{2}$.

Therapies are modelled changing parameters and maintaining the total dose constant:

$$
\begin{gathered}
\mathrm{Ch}=\int_{T_{i}}^{T_{f}} g(t) \mathrm{d} t=\int_{T_{i}}^{T_{f}}\left[r_{i}-f_{i}(t)\right] \mathrm{d} t, \\
\mathrm{Im}=\int_{T_{i}}^{T_{f}} h(t) \mathrm{d} t=\int_{T_{i}}^{T_{f}}\left[\kappa_{i}(t)-c_{i}\right] \mathrm{d} t,
\end{gathered}
$$

where $\left[T_{i}, T_{f}\right]$ is the time interval of administration of the treatments and Ch refers to chemotherapy while Im to immunotherapy. Actual values of the total dose are set accordingly to [29]: $\mathrm{Ch}=9.66$ and $\mathrm{Im}=971$. 
TABLE 1. Table of the parameters and initial conditions used in all the simulations.

\begin{tabular}{ccccc}
\hline Parameter & Unit & Value/Range & Interpretation & Source \\
\hline$r_{1}$ & days $^{-1}$ & 0.15 & Tumor growth rate of $x_{1}$ & {$[7]$} \\
$r_{2}$ & days $^{-1}$ & 0.11 & Tumor growth rate of $x_{2}$ & {$[7]$} \\
$b_{i j}$ & days $^{-1}$ & $0.01-1$ & Intratumoral competition & Estimated \\
$c_{i}$ & days $^{-1}$ & 5.5 & Killing rate by the immune system & {$[16]$} \\
$K_{i}$ & cells & $5 \times 10^{7}$ & Tumor carrying capacity & {$[7,16]$} \\
$H$ & cells & $3 \times 10^{5}$ & Immune system threshold & {$[16]$} \\
$\alpha_{i}$ & days $^{-1}$ & $6 \times 10^{-9}$ & Immune recruitment rate & {$[16]$} \\
$\beta$ & days $^{-1}$ & 0.8 & Immune system growth rate & Estimated \\
\hline
\end{tabular}
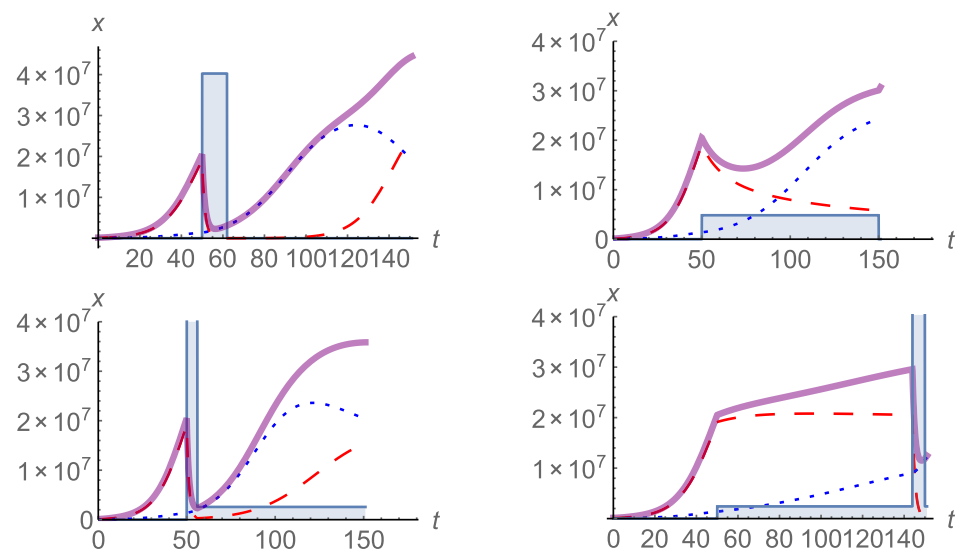

Figure 1. Cancer populations time courses for configuration $C O E$ under chemotherapy. The dotted blue line and the dashed red line denote, respectively, $x_{1}$ and $x_{2}$, and the continuous violet line corresponds to $x(t)=x_{1}(t)+x_{2}(t)$. Vertical blue segments indicate times of drug administration. Top left panel: "bang" therapy at the beginning of the treatment window $W$ $(I=0.049)$. Top right panel: continuous protocol $(I=0.069)$. Bottom left panel: switch to pulse with initial "bang" $(I=0.066)$. Bottom right panel: switch to pulse with final "bang" $(I=0.136)$.

\subsection{Classical protocols}

We start by considering some "classical" chemotherapy protocols, [32], namely a "bang" or maximum tolerated dose (MTD, the dose is administered in a relatively short interval), a continuous protocol (where the same dose is given each day of the treatment window $W$ ), and finally, the switch to pulse in which a "bang" is preceded, or followed, by a continuous protocol.

Results, displayed in Figure 1, confirm previous finding [26, 27] that, for heterogeneous tumors (as in the $C O E$ case), in general chemotherapy alone is not efficient, due to clonal inversion and emergence of resistant subpopulations. However, a switch to pulse protocol consisting of a continuous therapy followed by a final bang yields better results, with an index value markedly larger than the others. This result is due to the fact that the dominant clone $x_{1}$ controls the resistant one $x_{2}$ and most of the total cancer load is made of $x_{1}$ when the final bang is applied. These results are coherent with findings proposed in [28].

\subsection{Splitting the dose}

In order to further investigate the effectiveness of chemotherapy protocols, different time courses with a given constant dose are simulated. In other words, the total dose is kept fixed in a given treatment window $W$, while it 

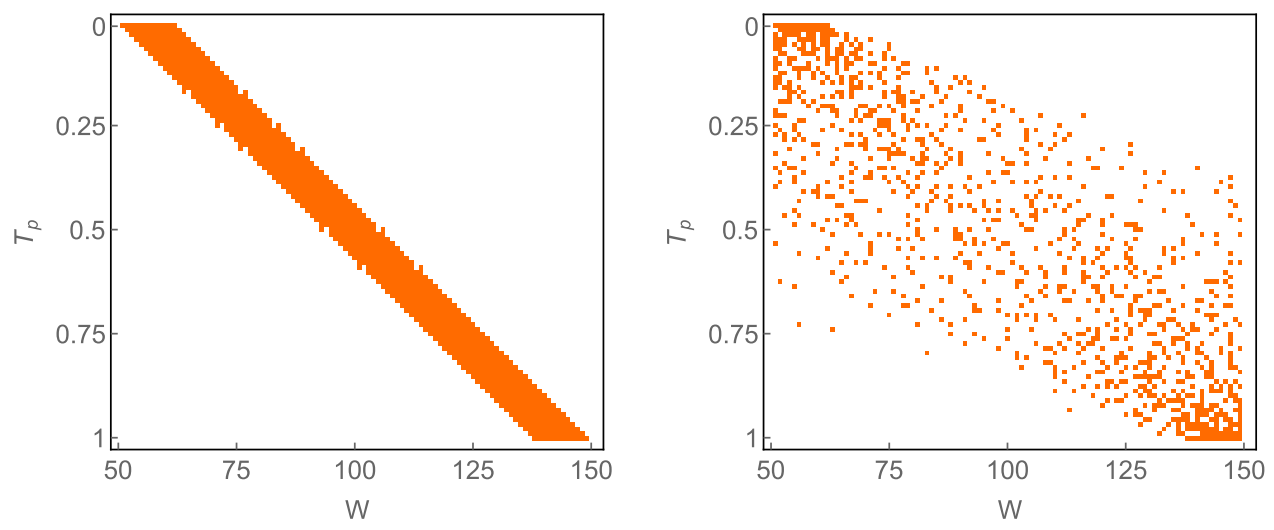

Figure 2. Protocols with $n=12$ "on" days of treatment (red dots) distributed, for increasing values of $T_{p}$ on consecutive days (left panel) or at random in the window $W$ (right panel). Vertical axis reports values of $T_{p}$ for each protocol while the horizontal axis indicates the days of the treatment window.
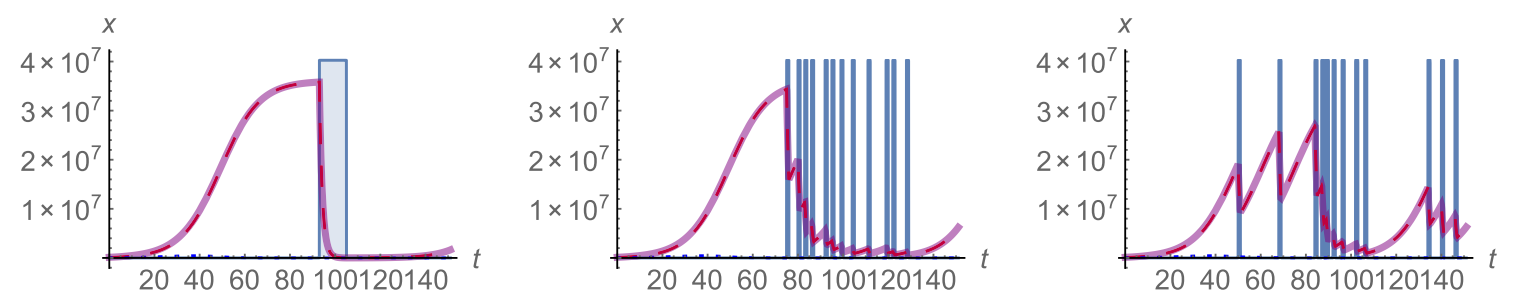

Figure 3. Cancer populations time courses for configuration EC1 under chemotherapy. The dotted blue line and the dashed red line denote, respectively, $x_{1}$ and $x_{2}$, and the continuous violet line corresponds to $x(t)=x_{1}(t)+x_{2}(t)$. Vertical blue segments indicate times of drug administration. Days of treatment are chosen with an average at the middle of $W\left(T_{p}=0.5\right)$. Left panel: 12 consecutive with $I=0.417$. Central and right panel: 2 realizations of random administration, here $I=0.452$ for both panels.

is varied how this dose is subdivided in different days and how they are distributed. In the sequel the treatment window is chosen in the time interval from $T_{i}=50$ to $T_{f}=150$, i.e. $W$ is 100 days long. Here, it is assumed that, in this interval, treatment is administered in $n=12$ days: a day of treatment is called an "on" day. These $n$ days can be consecutive or distributed at random in $W$. Thus, protocols may differ in the starting day and in the temporal distribution of "on" days. These differences are captured by introducing a translated average drug administration time $T_{p} \in[0,1]$, such that $T_{p}=0$ if "on" days are consecutive starting from $T_{i}$ and $T_{p}=1$ if "on" days are again consecutive terminating in $T_{f}$. Obviously, in general to the same value of $T_{p}$ there may correspond different distributions of "on" days. The left panel of Figure 2 displays, for a set of increasing values of $T_{p}$, a realization of the distribution of "on" days. On the left, "on" days are consecutive, and there is just one possible distribution, while on the right they are randomly distributed.

Figure 3 shows applications of three chemotherapy protocols corresponding to the same $T_{p}=0.5$, but differing in the distribution of "on" days. Here the clonal configuration is EC1. In the leftmost panel all dose is concentrated in $n=12$ successive days such that its middle value is just at the center of treatment window $W$. In the central and rightmost panels, the "on" days are random with $T_{p}=0.5$, i.e. their distribution is spread around a middle point of $W$. Comparison with the previous case shows that now, for each protocol, all index $I$ values are much larger and very close to each other. An explanation is that, in this condition of competitive exclusion, emergence of resistance is a negligible effect and that the sensitive clone $x_{1}$ is curtailed by the therapy. 

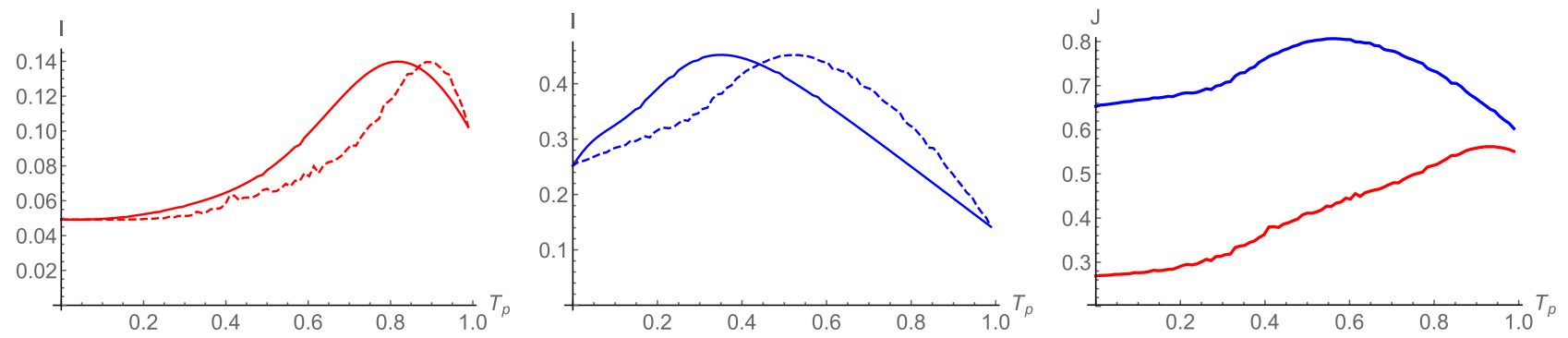

Figure $4 . I$ is plotted as function of $T_{p}$ for the $C O E$ configuration (left panel) and for the $E C 1$ case (central panel). The continuous line represents the outcome of a bang protocol with consecutive "on" days and the dashed line is the average value of $I$ for different simulations with the same $T_{p}$. In the right panel the index $J$ is plotted as a function of $T_{p}$. Red and blue lines refer to the $C O E$ and $E C 1$ configurations, respectively, under protocol with consecutive "on" days.

The closeness of index values supports the idea that what matters is the value of the shifted average $T_{p}$, rather than the actual distribution of "on" days.

Note that the index $T_{p}$ is more general than starting time of a protocol. In the case of distributed protocols, the starting time of the treatment could be the same for different protocols with different averaged $T_{p}$ and results shown in Figure 2 suggest that the value of the average $T_{p}$ is more important than the actual distribution of "on" days. Moreover, in the case of consecutive "on" days, as shown in left panel of Figure 3, the index $T_{p}$ corresponds, apart from a common temporal shift, to the starting time.

The comparison of different protocols has been generalized by considering increasing values of $T_{p}$ and, for each such value, generating different (17) random realizations of "on" days distributions; for each $T_{p}$ the corresponding average of $I$ is computed. The efficiency index is plotted, as a function of $T_{p}$ in Figure 4, for configurations $C O E$ (left) and $E C 1$ (central), respectively. The continuous curve is the index $I$ for distributions of all consecutive days, and the dashed one is the average of random distributions.

As shown in the left panel of Figure 4, in the COE state, $I$ is in general small and varies little in the range of its possible values i.e. [0,1]; however, chemotherapy is slightly more efficient when administered toward the end of the treatment window and that confirms and generalizes results reported above (compare Fig. 1 and related comments in the text).

By contrast, in the EC1 case (central panel of Fig. 4), the therapy is, in general, efficient and the best results are obtained for protocols corresponding to values of $T_{p}$ in the middle of the range $[0,1]$. In $E C 1$ the sensitive population tends, not just to control, but to suppress the resistant clone $x_{2}$ and so the problem of the resistance is less acute; rather it is important to avoid an uncontrolled growth of the sensitive population $x_{1}$. Thus the therapy should be administered earlier, to avoid $x_{1}$ becoming too large but not before it has almost eliminated $x_{2}$. Obviously, the $T_{p}$ values corresponding to the maximum $I$, depend in general also on the initial conditions and on the ratio of sensitive versus resistant populations. As a general rule, it would appear that the larger is the competitive advantage of the sensitive population the earlier the therapy should be applied.

As remarked earlier, the same configuration (COE or EC1) may correspond to different degrees of competition. Actual values of $I$ depend on these competition levels and so the curve representing a case in which $E C 1$ is relatively weak would resemble a curve relative to $C O E$ more closely than when competition is very strong.

In order to compare index $I$ with others proposed in the literature, we consider an index similar to $H$, see equation (3.1), namely

$$
J=\frac{1}{2}\left[\left(1-\frac{x_{\mathrm{post}}}{K}\right)+\left(1-\frac{\int_{T_{i}}^{T_{f}} x(t) \mathrm{d} t}{\int_{T_{i}}^{T_{f}} x_{c}(t) \mathrm{d} t}\right)\right],
$$



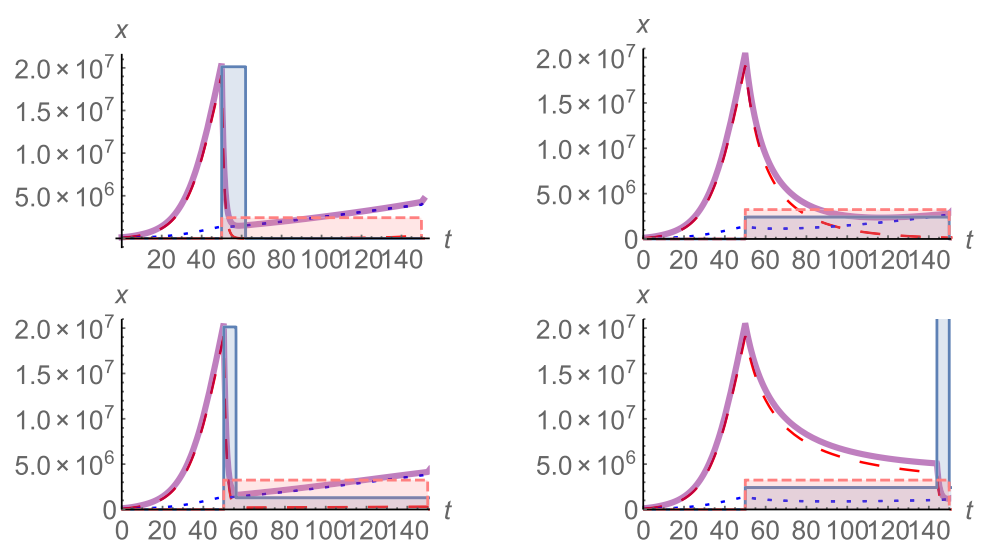

Figure 5. Combination therapy. A metronomic immunotherapy is administered in addition to the classical chemotherapic protocols of Figure 1, in case of configuration $C O E$. The pink horizontal bar represents immunotherapy, otherwise notations are the same as Figure 1. Indices are $I=0.543$ for an initial bang, $I=0.552$ for a metronomic protocol, $I=0.544$ for the switch to pulse with an initial bang and $I=0.533$ for switch to pulse the one with a final bang.

where $K=K_{1}+K_{2}$, which, differently from $H$, is such that a higher efficiency corresponds to larger $J$ values and all weights are chosen equal to $1 / 2$, so that $J$ is in the $[0,1]$ range, making the comparison with $I$ easier. Furthermore, here the total amount of drug is considered fixed in the treatment window and hence in (4.2) it does not appear as a variable.

Values of $J$, as a function of $T_{p}$, are shown in the right panel of Figure 4 for $C O E$ and $E C 1$ respectively. Despite obvious differences in numerical values, the indices $I$ and $J$ share the same trend: in $C O E$ they are slowly increasing with a maximum toward the end of the temporal range whereas for $E C 1$ their time course is characterized by a maximum at intermediate values of $T_{p}$.

\subsection{Combination therapy}

The advantages of using a combination therapy are so well documented in the literature see e.g. [17, 33], to have become commonplace. We replicate here, for combination of chemo- and immunotherapy, the simulations carried out for the "classical" protocols, investigated in Section 4.1, by adding to chemotherapy a continuous immunotherapy. Results are shown in Figure 5: as expected the index $I$ is larger than in case of chemotherapy alone. Now both clones are curtailed by therapies, hence the timing is less relevant; indeed the index $I$ is virtually the same in all cases.

In the line of Section 4.2, we investigate the effectiveness of combination therapies, by simulating protocols spanning the range of $T_{p}$. The graphs of Figure 6 show that the increment of $I$, with respect to chemotherapy alone, is especially marked for $C O E$, even though $E C 1$ is the configuration for which the treatment is more efficient (see left panel of Fig. 6) and $I$ has small variations across trials with the same $T_{p}$ (compare right panel of Fig. 6). Interestingly, the largest $I$ values arise when the chemotherapy is administered early and this is in contrast with what happens for chemotherapy alone (compare left and central panels of Fig. 4). The reason is that now the resistant cells are contrasted not just by the sensitive ones but also by the action of the immune system, enhanced by immunotherapy, hence their growth becomes less relevant and the earlier chemotherapy is applied, the better.

This result is coherent with the idea, put forward in the previous subsections, that the greater the selective pressures, the earlier should be the treatment. It should be noted that the case of early bang (MTD) of chemotherapy, coupled with a continuous immunotherapy is also advocated, as best treatment strategy, on the basis of a formal optimization procedure in [18] and references therein. Thus, the present analysis does not just 

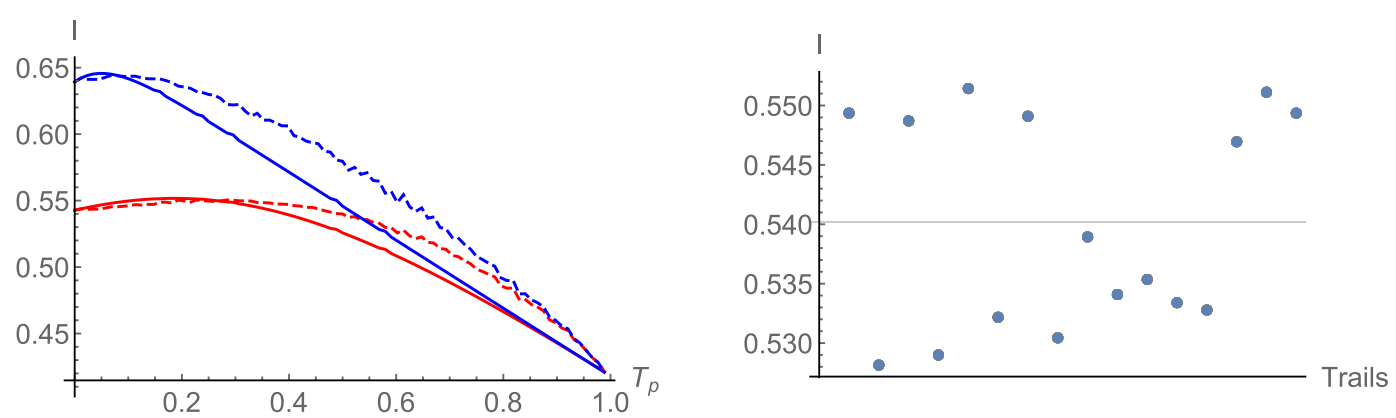

Figure 6. Left panel: $I$ vs. $T_{p}$, in the case of combination therapy. Notations are the same as Figure 4 and red lines correspond to $C O E$ and blue lines to $E C 1$ configurations, respectively. The continuous line represents the outcome of protocol with bang of 12 consecutive "on" days and the dashed lines are the average value of $I$ for 17 simulations with the same $T_{p}$ in the $C O E$ and $E C 1$ configurations. Right panel shows an example of the different simulations generated, and then averaged, for a specific $T_{p}=0.5$ in the $C O E$ configuration.
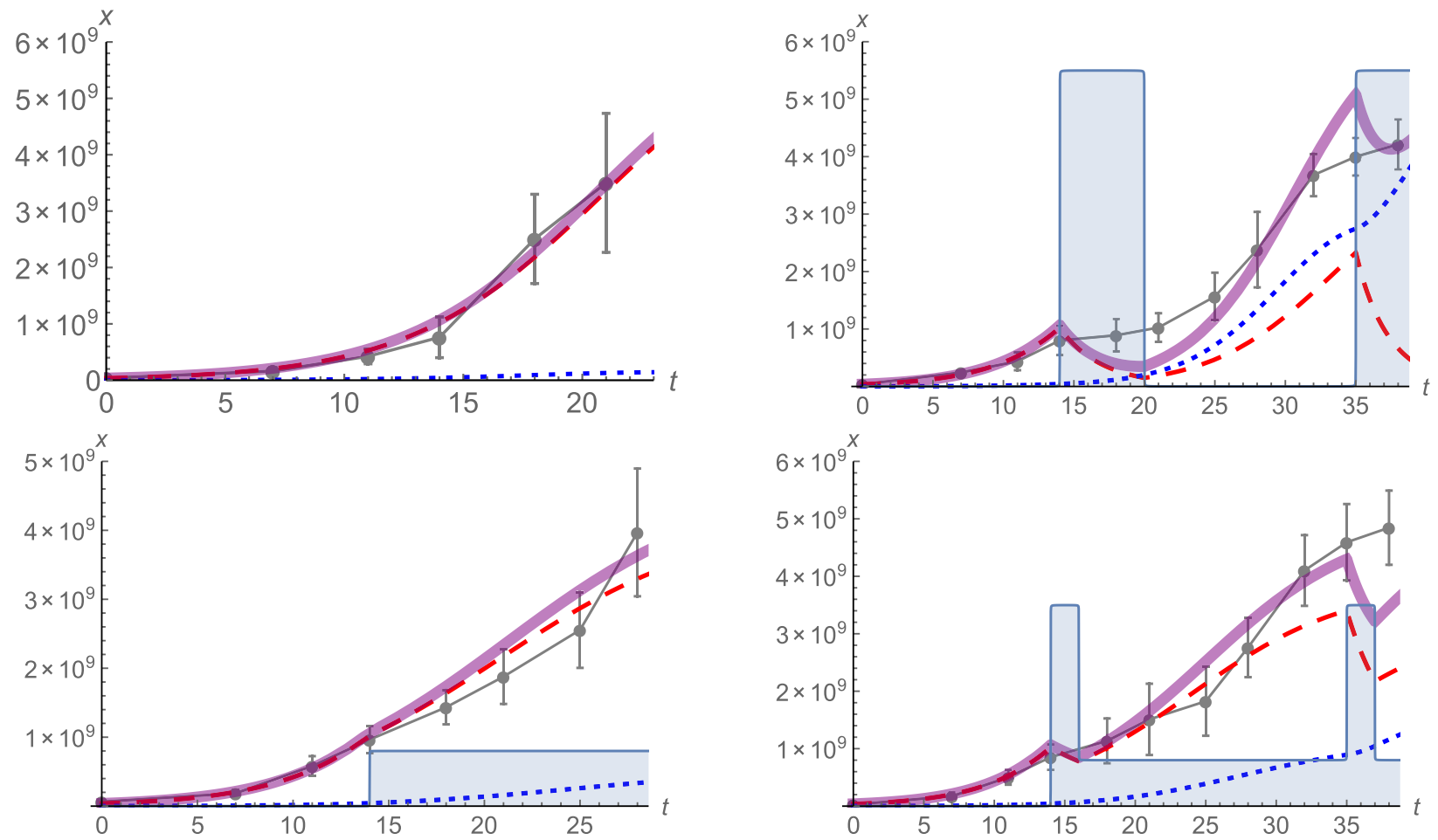

Figure 7. Comparison with experimental data: panels refer to control (top left), maximum tolerated dose (bangs), continuous low dose (bottom left), switch to pulse (bottom right). The grey line denotes the experimental results and the other notations are as in Figure 1. Index values are, from top right to bottom right panels, $I=0.001 ; I=0.009 ; I=0.015$.

confirms the findings of previous studies but it adds an important information on the timing of treatments in relation to clonal composition. 

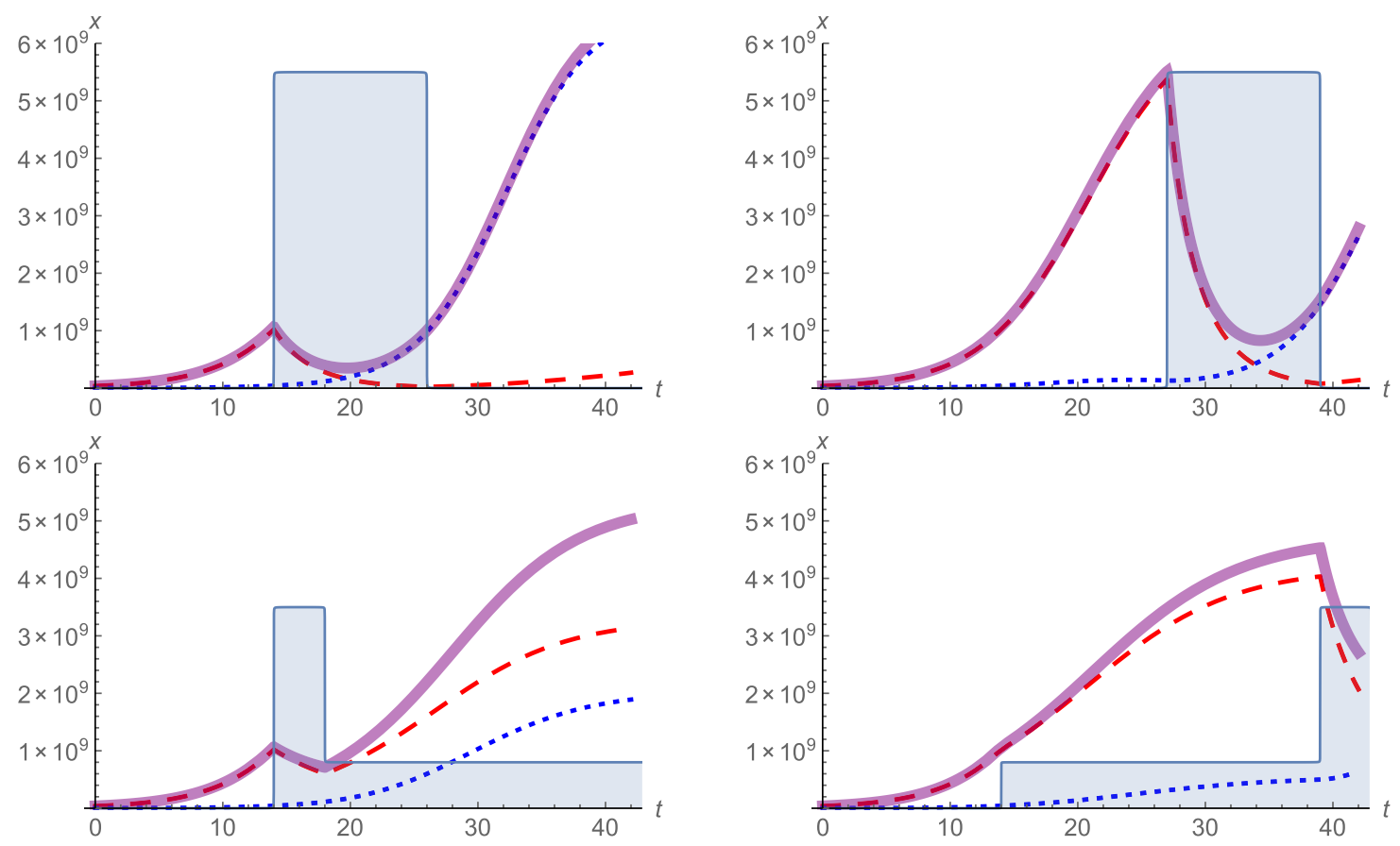

Figure 8. New protocols tested in silico with the same settings of biological data and notations of Figure 7. Top panels refers to initial bang (left panel, $I=0.000, T_{p}=0$ ) and final one (right panel, $\left.I=0.073, T_{p}=1\right)$. Bottom panels propose switch to pulse protocols with initial bang $(I=0.004)$ and final bang $(I=0.009)$.

\subsection{Comparison with experimental data}

In order to test the validity of our analysis and conjecture on the effectiveness of different protocols, we have considered data from an experiment reported in [32]. Breast tumor cells were transplanted in mice and treated with different schedules of chemotherapy. Figure 7 shows the time course of the data, for different experimental protocols, together with the predictions of our model. The control case is displayed in the top left panel and the top right panel shows the results for two "bangs" at the beginning and at the end of the treatment window. Bottom panels refer to the case of continuous therapy and switch to pulse with initial and final "bangs".

Parameters and initial conditions are set as before apart from: $r_{1}=0.25 ; r_{2}=0.29 ; b_{12}=0.1 ; b_{21}=0.5, K_{1}=$ $K_{2}=10^{10} ; H=3 \times 10^{6}$. Initial conditions now are set as: $x_{1}(0)=3.7 \times 10^{7}, x_{2}(0)=1 \times 10^{6}, z(0)=5 \times 10^{5}$. Parameters are set in a EC1 configuration, however the considered numbers of cells are such that the effects of inter-species competition are small. Chemotherapies are modelled changing parameters as follows: "bangs" of 6 "on" days with $r_{1}-g_{1}=-0.3$, continuous low dose with $r_{1}-g_{1}=0.17$, switch to pulse with "bangs" of 2 days where $r_{1}-g_{1}=-0.1$ and a continuous low dose $r_{1}-g_{1}=0.17$.

Note the good agreement between the data and the time courses of cancer population predicted by our model. Moreover our simulations allow to gain some information on the acquired resistance after the treatment window. For instance comparing two bangs (top right panel of Fig. 7) with a metronomic treatment (bottom left panel), it is clear that even though the final number of tumoral cells is comparable, the first therapy has generated a population made up mostly of resistant cells, while metronomic schedule reduces the emergence of resistance, coherently with reports in literature, see e.g. [1]. It is apparent from the data that all proposed therapies are quite ineffectual, and, this situation is accurately predicted by the values of the index $I$ which are in any case very low. For comparison, we have computed the index $J$, equation (4.2), for the cases of maximum tolerated dose, $J=0.55$, and continuous therapies, $J=0.43$, (panels top right and bottom left of Fig. 7 , respectively). 
This indicates that $J$ gives an optimistic estimate of the effect of the therapy on cancer growth, as it fails to take explicitly into account the emergence of the resistant population. Furthermore, the values of $J$ are very close, whereas the corresponding values of $I$ differ almost of an order of magnitude, showing greater effectiveness of discrimination.

Obviously, many other protocols can be adopted, and to investigate which could give better results, we simulate the performance of new protocols obtained by changing the timing of "bangs" and switch to pulse treatments. Results are shown in Figure 8 from which it is clear that the best outcome is for a late bang (see top right panel) as also proposed in [28]. For this case, index $I$ is much larger than values yielded by experimental protocols.

\section{Conclusion}

Results presented in this paper clearly confirm previous findings [26, 27] on the relevance of inter-species competition among cancer cells in determining the outcome of medical treatment of cancer, coherently to biological findings, e.g. [35]. Differently from [26, 27], where different amounts of the total dose were considered, here the focus of the analysis has been on the distribution of the "on" days across a therapy window, while the total amount of drug has been kept fixed. The present analysis provides important new information on how the timing of the most efficient treatments is related to clonal composition.

A general rule seems to hold; when the environment is harsh and strong and the selective pressures lead to competitive exclusion with survival of the fittest clones, an early therapy appears to be better. For instance a harsh environment occurs when combination therapies are applied and this is indeed the case in which earliest therapies perform better.

On the contrary, when competitive exclusion does not apply, as for instance in a mild environment, the tumor is heterogeneous and the timing of protocols is less relevant, even though a relatively late therapy seems to be slightly more efficient as also proposed in [28].

Several studies highlight the relevance of microenvironment in affecting the fitness of tumor cells, changing the overall behaviour in terms of growth and response to therapy, see for instance [24]. Among others, certain factors such as hypoxia and acidosis, can be identified as hallmarks of a harsh environment, even though, unfortunately, it would be naive to expect a clear-cut classification of the type of microenvironment. However, the idea that the therapy should take into account this specificity is consistent with recent therapeutical approaches that not only target cancer cells but also aim to modulate the microenvironment, e.g. hypoxia activate prodrugs, [23] and blood vessel normalization [22].

A new index has been proposed, which compared with traditional indices has the property to take into account explicitly the cancer clonal composition and, in particular, the numerosity of resistant cell. Thus, in situations in which clonal switch leads to the emergence of drug resistance, the index proposed here has shown to be more suitable to capture this phenomenon. On the other hand, a limit of this index is that it requires, to be computed, precise clinical data on the composition of the different clonal populations.

Predictions of the model have been compared with data from an experiment on breast cancer where different time protocols were tested. A good agreement is found with the experimental time courses of cancer load, both in the control case and under different therapies. Then, new protocols have been proposed and simulated and, at least one of them performs better in silico than those used in the experiments.

Acknowledgements. This work is supported by the "Departments of Excellence 2018 - 2022" Grant awarded by the Italian Ministry of Education, University and Research (MIUR) (L. 232/2016) CUP: E11G18000350001.

\section{REFERENCES}

[1] N. André, M. Carré and E. Pasquier, Metronomics: towards personalized chemotherapy? Nat. Rev. Clin. Oncol. 11 (2014) 413-431.

[2] N. Bellomo, N. Li and P.K. Maini, On the foundations of cancer modelling: selected topics, speculations, and perspectives. Math. Models Methods Appl. Sci. 18 (2008) 593-646. 
[3] A. Besse, G. Clapp, S. Bernard, F. Nicolini, D. Levy and T. Lepoutre, Stability analysis of a model of interaction between the immune system and cancer cells in cml. Bull. Math. Biol. 80 (2017) 1084-1110.

[4] S. Bunimovich-Mendrazitsky, H. Byrne and L. Stone, Mathematical model of pulsed immunotherapy for superficial bladder cancer. Bull. Math. Biol. 70 (2008) 2055-2076.

[5] C. Carrère, Optimization of an in vitro chemotherapy to avoid resistant tumours. J. Theor. Biol. 413 (2017) $24-33$.

[6] L.G. De Pillis and A. Radunskaya, A mathematical tumor model with immune resistance and drug therapy: an optimal control approach. Comput. Math. Methods Med. 3 (2001) 79-100.

[7] L.G. De Pillis, W. Gu and A.E. Radunskaya, Mixed immunotherapy and chemotherapy of tumors: modeling, applications and biological interpretations. J. Theor. Biol. 238 (2006) 841-862.

[8] V.T. DeVita and P. S. Schein, The use of drugs in combination for the treatment of cancer: rationale and results. New England J. Med. 288 (1973) 998-1006.

[9] A. D'Onofrio, U. Ledzewicz and H. Schättler, On the dynamics of tumor-immune system interactions and combined chemo-and immunotherapy, in New Challenges for Cancer Systems Biomedicine. Springer, Berlin (2012) 249-266.

[10] R. Eftimie, J.L. Bramson and D.J. Earn, Interactions between the immune system and cancer: a brief review of non-spatial mathematical models. Bull. Math. Biol. 73 (2011) 2-32.

[11] F. Frascoli, P.S. Kim, B.D. Hughes and K.A. Landman, A dynamical model of tumour immunotherapy. Math. Biosci. 253 (2014) 50-62.

[12] R.A. Gatenby, J. Brown and T. Vincent, Lessons from applied ecology: cancer control using an evolutionary double bind. Cancer Res. 69 (2009) 7499-7502.

[13] M. Gerlinger and C. Swanton, How darwinian models inform therapeutic failure initiated by clonal heterogeneity in cancer medicine. Br. J. Cancer 103 (2010) 1139-1143.

[14] N. Hartung, C.T.-K. Huynh, C. Gaudy-Marqueste, A. Flavian, N. Malissen, M.-A. Richard-Lallemand, F. Hubert and J.-J. Grob, Study of metastatic kinetics in metastatic melanoma treated with b-raf inhibitors: introducing mathematical modelling of kinetics into the therapeutic decision. PloS One 12 (2017) e0176080.

[15] N.L. Komarova, J.A. Burger and D. Wodarz. Evolution of ibrutinib resistance in chronic lymphocytic leukemia (cll). Proc. Natl. Acad. Sci. 111 (2014) 13906-13911.

[16] V.A. Kuznetsov, I.A. Makalkin, M.A. Taylor and A.S. Perelson, Nonlinear dynamics of immunogenic tumors: parameter estimation and global bifurcation analysis. Bull. Math. Biol. 56 (1994) 295-321.

[17] H. Ledford, The perfect blend. Nature 532 (2016) 162-164.

[18] U. Ledzewicz and H. Schättler, Application of mathematical models to metronomic chemotherapy: What can be inferred from minimal parameterized models? Cancer Lett. 401 (2017) 74-80.

[19] U. Ledzewicz, S. Wang, H. Schättler, N. André, M.A.H. Heng and E. Pasquier, On drug resistance and metronomic chemotherapy: a mathematical modeling and optimal control approach. Math. Biosci. Eng. 14 (2017) $217-235$.

[20] K. Leon, K. Garcia, J. Carneiro and A. Lage, How regulatory cd25+cd4+t cells impinge on tumor immunobiology? On the existence of two alternative dynamical classes of tumors. J. Theor. Biol. 247 (2007) 122-137.

[21] A. Lorz, T. Lorenzi, M.E. Hochberg, J. Clairambault and B. Perthame, Populational adaptive evolution, chemotherapeutic resistance and multiple anti-cancer therapies. ESAIM: M2AN 47 (2013) 377-399.

[22] J.D. Martin, G. Seano and R.K. Jain, Normalizing function of tumor vessels: Progress, opportunities, and challenges. Ann. Rev. Physiol. 81 (2019) 505-534.

[23] F. Meng, J.W. Evans, D. Bhupathi, M. Banica, L. Lan, G. Lorente, J.-X. Duan, X. Cai, A.M. Mowday, C.P. Guise, et al., Molecular and cellular pharmacology of the hypoxia-activated prodrug th-302. Mol. Cancer Ther. 11 (2012) 740-751.

[24] S.M. Mumenthaler, J. Foo, N.C. Choi, N. Heise, K. Leder, D.B. Agus, W. Pao, F. Michor and P. Mallick, The impact of microenvironmental heterogeneity on the evolution of drug resistance in cancer cells. Cancer Inform. 14 (2015) 19-31.

[25] J.D. Murray, Mathematical Biology. Springer-Verlag, Berlin (2002).

[26] E. Piretto, M. Delitala and M. Ferraro, Combination therapies and intra-tumoral competition: insights from mathematical modelling. J. Theor. Biol. 446 (2018) 149-159.

[27] E. Piretto, M. Delitala and M. Ferraro, How combination therapies shape drug resistance in heterogeneous tumoral populations. Lett. Biomath. 5 (2018) S160-S177.

[28] C. Pouchol, J. Clairambault, A. Lorz and E. Trelat, Asymptotic analysis and optimal control of an integro-differential system modelling healthy and cancer cells exposed to chemotherapy. J. Math. Pures. Appl. 116 (2018) 268-308.

[29] R. Ramakrishnan, D. Assudani, S. Nagaraj, T. Hunter, H.-I. Cho, S. Antonia, S. Altiok, E. Celis and D.I. Gabrilovich, Chemotherapy enhances tumor cell susceptibility to ctl-mediated killing during cancer immunotherapy in mice. J. Clin. Investig. 120 (2010) 1111.

[30] N.A. Saunders, F. Simpson, E.W. Thompson, M.M. Hill, L. Endo-Munoz, G. Leggatt, R.F. Minchin and A. Guminski, Role of intratumoural heterogeneity in cancer drug resistance: molecular and clinical perspectives. EMBO Mol. Med. 4 (2012) 675-684.

[31] R. Serre, S. Benzekry, L. Padovani, C. Meille, N. Andre, J. Ciccolini, F. Barlesi, X. Muracciole and D. Barbolosi, Mathematical modeling of cancer immunotherapy and its synergy with radiotherapy. Cancer Res. 76 (2016) 4931-4940.

[32] Y. Shaked, U. Emmenegger, G. Francia, L. Chen, C.R. Lee, S. Man, A. Paraghamian, Y. Ben-David and R.S. Kerbel, Lowdose metronomic combined with intermittent bolus-dose cyclophosphamide is an effective long-term chemotherapy treatment strategy. Cancer Res. 65 (2005) 7045-7051.

[33] S. Slovin, Chemotherapy and immunotherapy combination in advanced prostate cancer. Clin. Adv. Hematol. Oncol. 10 (2012) 90-100. 
[34] T. Stiehl, C. Lutz and A. Marciniak-Czochra, Emergence of heterogeneity in acute leukemias. Biol. Direct 11 (2016) 51.

[35] D.P. Tabassum and K. Polyak, Tumorigenesis: it takes a village. Nature Rev. Cancer 15 (2015) 473-483.

[36] S. Wilson and D. Levy, A mathematical model of the enhancement of tumor vaccine efficacy by immunotherapy. Bull. Math. Biol. 74 (2012) 1485-1500. 